\title{
Commentary: Hormones, Diet, and Botanicals
}

\author{
Jerome Engel, Jr.,* and Solomon L. Moshé ${ }^{\dagger}$ \\ *Departments of Neurology, Neurobiology, and Psychiatry \& Biobehavioral Sciences and the Brain Research Institute, David \\ Geffen School of Medicine at UCLA, Los Angeles, California 90095-1769, and Department of Neurology, Albert Einstein \\ College of Medicine, Bronx, New York 10461
}

\begin{abstract}
Summary: In contrast to the high technology-driven interventions discussed in the previous presentations, hormonal therapy, diet, and botanicals are natural interventions that make use of homeostatic or traditional approaches. Clear advantages of
\end{abstract}

these largely adjunctive therapies are discussed, as well as unique obstacles to bringing these alternative interventions into the mainstream. Key Words: Epilepsy, therapy, hormones, ketogenic diet, botanicals.
The articles in this section diverge considerably from most articles in the rest of this issue, which deal largely with high technology-driven interventions, many of which are invasive. In contrast, hormonal therapy, diet, and botanicals are natural interventions that make use of homeostatic or traditional approaches. Despite obvious advantages to pursuing these relatively inexpensive treatments, it has proven difficult- because there are no patentable devices or products that can be commercially exploited - to obtain adequate funding for the expensive clinical trials that are usually necessary to prove effectiveness. It is a sad comment indeed on our system of healthcare that profit calculations so often determine the direction of biomedical research.

Currently practiced hormonal and diet therapies are adjunctive, however, and are not intended to replace antiepileptic drug treatment completely. Thus, these complementary approaches cannot be compared with the more aggressive treatments discussed elsewhere in this issue, some of which do have the potential for eliminating seizures, as well as the need for standard pharmacotherapy. Large clinical trials are still needed to establish exactly how effective hormonal therapy and diets are as adjunctive treatments for epileptic seizures, and also for defining the specific patient populations for whom these treatments would be most appropriate, for clearly delineating their risks and benefits, and for ultimately eluci-

Address correspondence and reprint requests to: Jerome Engel, Jr., M.D., Ph.D., Department of Neurology, David Geffen School of Medicine at UCLA, 710 Westwood Plaza, Los Angeles, CA 90095-1769.

E-mail: engel@ucla.edu. dating the underlying mechanisms, which could lead to safer and more effective means of achieving the same neurobiological effects.

Hormonal therapy is often proposed as a form of antiseizure medication and has been discussed mostly in terms of catamenial epilepsy. However, hormonal therapy (e.g., adrenocorticotropic hormone) has been used as the primary medical therapy for infantile spasms. ${ }^{1}$ The role of gonadal hormones on brain development and epilepsy is actively explored, especially their effect on the maturation of GABAergic systems involved in seizure control, including the age-specific-modulation of $\mathrm{GABA}_{\mathrm{A}}$ receptors and their transition from depolarizing to hyperpolarizing effects. ${ }^{2,3}$ There are also studies investigating the possible neuroprotective effects of gonadal hormones on seizure-induced injury. ${ }^{4}$ These studies may be important for the identification of sex-specific treatments and neuroprotective strategies. ${ }^{5}$

It appears that the ketogenic diet is in much the same situation that epilepsy surgery was some 8 years ago, before the landmark randomized clinical trial of Wiebe et al. ${ }^{6}$ definitively demonstrated the effectiveness of surgical treatment for temporal lobe epilepsy. As a result, surgery was deemed the treatment of choice for medically refractory temporal lobe seizures by an official practice parameter issued by the American Academy of Neurology, the American Epilepsy Society, and the American Association of Neurological Surgeons. ${ }^{7}$ As is the case with those who practice the ketogenic diet today, prior to the Wiebe et al. ${ }^{6}$ study those who practiced epilepsy surgery knew that it worked, but it remained controversial because there were no class 1 data to sup- 
port the beneficial results reported in hundreds of published surgical series. Unfortunately, however, there is no evidence that the randomized controlled trial and practice parameter have made a significant difference in either the numbers of patients referred for surgery or the amount of money awarded for research to determine who should be referred for surgery, to further improve its safety and efficacy, or to reduce its cost.

If definitive results of clinical research are to have a substantial effect on patient care, studies also need to be conducted to determine how patients and physicians make decisions regarding therapeutic directions, particularly when alternative therapies are involved. It may be that much more can be achieved at this point by devising better ways to maximize patient access to their best options among all available treatments than by further research to identify new treatments.

Although research on botanicals could result in patentable compounds that would be of interest to the pharmaceutical industry, most of these herbal remedies are already available over the counter in health food stores. Because they do not come under the control of the Food and Drug Administration, and because their composition is not rigidly controlled, physicians often are reluctant to suggest them to their patients. To argue that we are long overdue in seriously investigating traditional and folk remedies is not heretical. Rather, it is a measure of the arrogance of modern medicine that complementary and alternative therapies are usually rejected out of hand. Fortunately, however, there is an increasing interest not only in botanicals, but also in practices, such as acupuncture, meditation, and ritual. Therapeutic interventions that have been deemed effective over thousands of years should not be ignored; they at least deserve rigorous experimental investigation. Conversely, more research is necessary to permit identification of potentially harmful effects on people with epilepsy of over-the-counter herbal remedies. ${ }^{10}$ Again, however, there is little financial support for such studies when there is no obvious profit to be made, even if findings are clinically relevant.

The beneficial results of complementary and alternative medicine in various cultures are usually dismissed as a placebo effect. Placebo effects, however, are not unique to traditional and folk medicine: they also account for a significant percentage of the improvement brought about by modern medical therapy, as is clearly demonstrated whenever clinical trials include a placebo control group. The placebo effect is not a statistical error-it is a real biological phenomenon that can have profound beneficial effects, and in some patients can even bring about a cure. ${ }^{8}$ Modern medicine must not denigrate and dismiss the placebo effect, but rather has an obligation to investigate why it occurs and to elucidate the underlying mechanisms. Understanding the pla- cebo effect could provide clues to powerful, inexpensive approaches to therapy.

Eighty percent of people with epilepsy live in developing countries, and of these, $80 \%$ receive no treatment at all, or inadequate treatment. ${ }^{9}$ In addition to cost, a major practical obstacle for epilepsy therapy in developing countries is assurance of consistent availability of antiepileptic drugs. Economic constraints and inadequate infrastructure prevent local pharmacies from maintaining adequate supplies of medications. In addition, in rural areas patients may need to walk several days to reach a clinic, and conditions such as health, weather, and social unrest often prevent them from obtaining their medication on a regular basis. Because the most commonly used antiepileptic drug by far, in developing countries, is phenobarbital, disruption of the prescribed regimen can result in withdrawal seizures and, at times, status epilepticus-which is worse for some patients than no treatment at all. There is, therefore, an overriding obligation to identify complementary and alternative medical approaches that are effective for controlling epileptic seizures in these populations, when these could be provided more consistently than modern drugs. Botanicals offer a particular advantage in this regard, because if specific plants prove to offer safe and effective antiepileptic therapy, families in rural areas of developing countries could be taught to grow their own antiepileptic drugs.

Perhaps the most difficult hurdle to overcome in developing safe and effective natural therapies for epilepsy, or any medical condition, remains adequate financial support. Modern biomedical research follows the money. Those with a vested interest in making a profit much more often determine what research is done than do the actual needs of people with illnesses such as epilepsy. There is no doubt that this has resulted in tremendous advances in small molecule pharmacotherapy, but other potentially effective approaches are being overlooked. This is particularly unfortunate for the large population of people with medical conditions who live in the developing world and are unable to take advantage of the costlier therapeutic developments that result from forprofit research efforts. As the dwindling of healthcare resources continues worldwide, we are increasingly dependent on philanthropic foundations to support research on developing relatively unprofitable but inexpensive and readily available therapies.

\section{REFERENCES}

1. Lux AL, Edwards SW, Hancock E, et al. The United Kingdom Infantile Spasms Study (UKISS) comparing hormone treatment with vigabatrin on developmental and epilepsy outcomes to age 14 months: a multicentre randomised trial. Lancet Neurol 2005;4: 712-717.

2. Galanopoulou AS. GABA receptors as broadcasters of sexually differentiating signals in the brain. Epilepsia 2005;46 Suppl 5:107112 
3. Galanopoulou AS. Dissociated gender-specific effects of recurrent seizures on GABA signaling in CA1 pyramidal neurons: role of GABA A $_{\text {A }}$ receptors. J Neurosci 2008;28:1557-1567.

4. Velíšková J, Velíšek, L. $\beta$-Estradiol increases dentate gyrus inhibition in female rats via augmentation of hilar neuropeptide Y. J Neurosci 2007;27:6054-6063.

5. Heida JG, Chudomel O, Galanopoulou AS, et al. Sex influence on the maturation of endogenous systems involved in seizure control. In: Takahashi T, Fukuyama Y, editors. Biology of seizure susceptibility in development of the brain. Progress in Epileptic Disorders Series. Montrouge, France: John Libbey Eurotext 2008:129-146.

6. Wiebe S, Blume WT, Girvin JP, Eliasziw M; Temporal Lobe Epilepsy Study Group. A randomized, controlled trial of surgery for temporal-lobe epilepsy. N Engl J Med 2001;345:311-318.
7. Engel J Jr, Wiebe S, French J, et al. Quality Standards Subcommittee of the American Academy of Neurology; American Epilepsy Society; American Association of Neurological Surgeons. Practice parameter: temporal lobe and localized neocortical resections for epilepsy [Erratum in: Neurology 2003;60:1396]. Neurology 2003;60:538-547.

8. Scott DJ, Stohler CS, Egnatuk CM, Wang H, Koeppe RA, Zubieta JK. Placebo and nocebo effects are defined by opposite opioid and dopaminergic responses. Arch Gen Psychiatry 2008;65:220-231.

9. Meinardi H, Scott RA, Reis R, Sander JWAS; ILAE Commission on the Developing World. The treatment gap in epilepsy: the current situation and ways forward. Epilepsia 2001;42:136-149.

10. Samuels N, Finkelstein Y, Singer SR, Oberbaum M. Herbal medicine and epilepsy: proconvulsive effects and interactions with antiepileptic drugs. Epilepsia 2008;49:373-380. 